\title{
Physiological Aspects of Soccer Refereeing Performance and Training
}

\author{
Carlo Castagna, ${ }^{1}$ Grant $A b t^{2}$ and Stefano D'Ottavio ${ }^{1}$
}

1 School of Sport and Exercise Sciences, Faculty of Medicine and Surgery, University of Rome Tor Vergata, Rome, Italy

2 Department of Sport, Health and Exercise Science, The University of Hull, Hull, UK

\section{Contents}

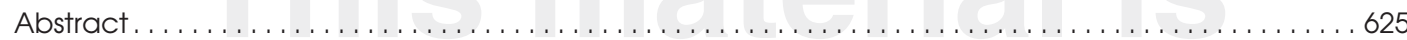

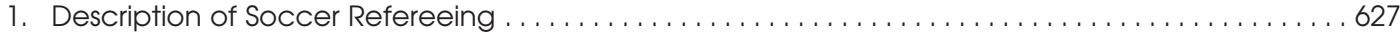

2. Match Performance................................................... 627

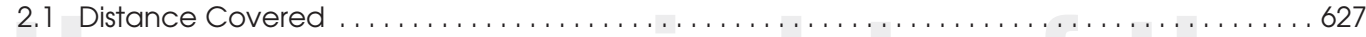

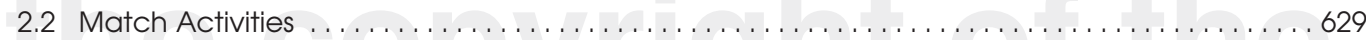

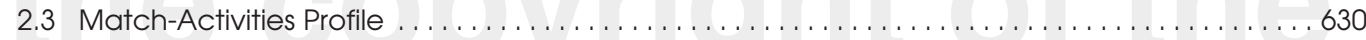

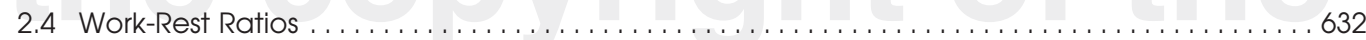

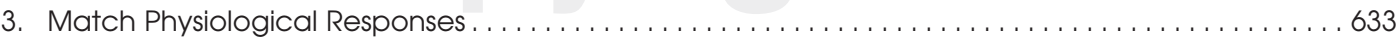

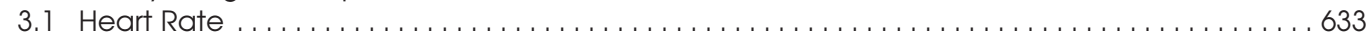

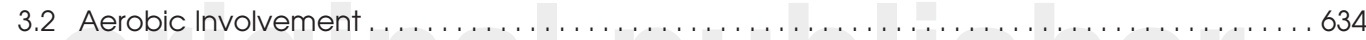

3.3 Blood Lactate . . . . . . . . . . . . . . . . . . . . . . . . . . . . . . . . . . . . . . . . . 636

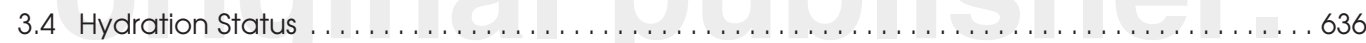

4. Physical Capacities of Soccer Referees . . . . . . . . . . . . . . . . . . . . . . . . . . . . . . . . . 638

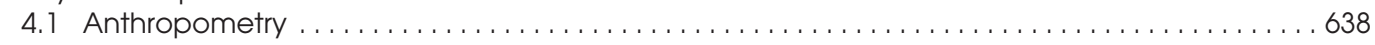

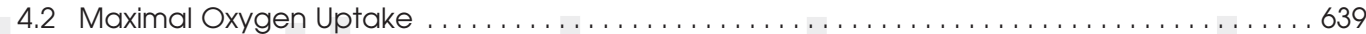

4.3 Anaerobic Performance ............................................. 639

4.4 Fitness Testing . . . . . . . . . . . . . . . . . . . . . . . . . . . . . . . . . . . . . . . . . . . . . . . . 640

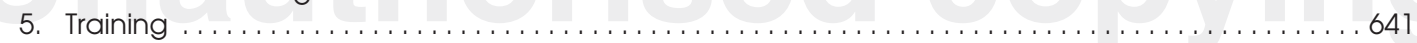

6. Mental Performance ...................................................... 643

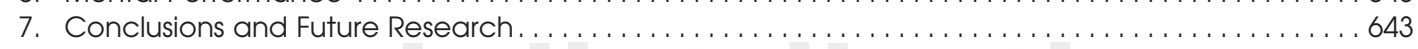

\section{Abstract}

The role of the referee is far from minimal in the economy of soccer, as very often, particularly in professional soccer, a wrong judgment may have profound implications on the outcome of the game. In this regard, a better knowledge of soccer refereeing can obviously benefit the game. Recent studies have shown that during a competitive match, an elite soccer referee may cover $9-13 \mathrm{~km}$ attaining approximately $85-90 \%$ and approximately $70-80 \%$ of maximal heart rate and maximal oxygen uptake $\left(\dot{\mathrm{V}}_{2 \max }\right)$, respectively. Of the total distance covered about $4-18 \%$ is covered at high intensity. Blood lactate concentration has been reported to be in the range of $4-5 \mathrm{mmol} / \mathrm{L}$; however, during competitive matches, blood lactate concentrations as high as $14 \mathrm{mmol} / \mathrm{L}$ have been observed. This figure is similar to that extensively reported for soccer players, specifically paralleling that observed in midfield players. However, compared with players, referees are 15-20 years older, often have a non-professional status and cannot be 


\begin{abstract}
substituted during the game. Furthermore, this important physical stress superimposes onto a high perceptual-cognitive workload throughout the entire game. In relation to fitness status, referees possess $\dot{\mathrm{V}}_{2}$ max values somewhat lower than the players they officiate, with mean values in the range of $44-50 \mathrm{~mL} / \mathrm{kg} / \mathrm{min}$. However, the methods used by the Federation Internationale de Football Association and the Union of European Football Associations to test referee fitness need to be changed as the current fitness tests do not relate to match performance. More task-specific tests such as the Yo-Yo Intermittent Recovery Test (YYIRT) have been devised and validated for use with referees. Given that aerobic performance is positively correlated with match performance, it is important that referees are trained to improve their ability to cover large distances during a match and also to repeat high-intensity efforts. A number of studies have shown large improvements in YYIRT performance following both short-term (12 weeks) and long-term (16 months) high-intensity interval training. Future research needs to focus on a number of important areas including the decision-making ability of referees when officiating under different conditions, such as high thermal strain, and the impact of age on both physical and mental performance.
\end{abstract}

Association football or soccer is considered the most popular sport currently played in the world. ${ }^{[1,2]}$

In 2003, around 220 million people were active members of the Federation Internationale de Football Association (FIFA), ${ }^{[1]}$ of which 150 million were active players (both male and female) at any competitive level. ${ }^{[3]}$ In order to depict a more comprehensive figure of the global soccer movement, a further 200-300 million of non-FIFA-affiliated active players should be taken into account. ${ }^{[1]}$ Consequently, it is estimated that there are around 400-500 million active soccer players worldwide.

During the last two decades, ${ }^{[2,4]}$ soccer has increasingly attracted the interest of researchers investigating the various aspects of this multifaceted sport. ${ }^{[4]}$ However, almost all the research efforts have been devoted to soccer players. Interest in the performance of soccer players can be seen by the number of reviews published. ${ }^{[4-14]}$

Every competitive soccer match must be regulated by a referee, two assistant referees and a side-line official (fourth official). It has been estimated ${ }^{[1]}$ that each week of a competitive season 1.3 million referees take to the football pitch with the aim of regulating the behaviour of the players and enforcing the rules of the game. Given the low frequency of scoring in a soccer match (approximately 2.7 goals per match), ${ }^{[15]}$ the decisions of a referee can have profound implications on the outcome of the game. With the introduction of the three points for a win rule and the increasing number of professional soccer teams being listed on the stock-exchange, it is easy to understand the importance of winning or losing in modern professional soccer. Consequently, soccer referees are progressively embracing fulltime status.

Despite the field-soccer referee being considered the 23 rd player of a soccer game, ${ }^{[16]}$ and their importance in ensuring that players uphold the laws of the game, very little scientific literature is available on soccer refereeing, especially compared with that available on players. However, interest in the scientific aspects of soccer refereeing has increased considerably during the last decade. For example, there were two scientific papers published in international peer-reviewed journals prior to 1995 , compared with 22 published between 1995 and the present time.

This article critically examines the current knowledge available on the performance-related aspects of soccer refereeing in an attempt to outline the 'state of the art' with reference to playing and training methods and accurate talent selection strategies. To ensure that we had examined all of the available 
empirical studies on soccer referees, we conducted a search on PubMed and SportDiscus databases using a number of issue-related search terms. This produced 150 studies related to soccer refereeing of which 30 were selected and discussed.

\section{Description of Soccer Refereeing}

Soccer is a tough combative sport where the players contest to gain possession of the ball. Nonetheless, contesting for the ball should be fair and sporting and it is the referee's role to regulate players' moves according to the laws of the game. ${ }^{[17]}$ The referee has full authority to enforce the laws of the game in connection with the match they have been appointed to ${ }^{[17]}$ with the decisions made by the referee being final. However, the referee may still change their decision on realising that it is incorrect. Changing a decision may be made on the advice of the assistant referee, but at the full discretion of the referee.

In order to regulate the rules of the game, the referee follows the playing action by moving inside the football pitch with no restriction. The referee's movements are made simply in an attempt to be in the best position for making a correct decision. The result of this is that the referee has to move constantly during the match and keep up with the game whatever the tempo. Furthermore, the referee has less freedom to pace their movements and cannot be substituted, except for an injury. Referees are further differentiated from players with respect to their average age. Recently Helsen and Bultynck ${ }^{[18]}$ reported that the field officials in charge of the final round of Union of European Football Associations (UEFA) 2000 Championship were an average age of $40.2 \pm 3.9$ years. This means that top-class referees $^{[18]}$ may be active at elite level while being almost double the average age of the players they officiate. ${ }^{[19]}$

Given that soccer referees are a vital element of the game, it is important that their training and performance be given due consideration by sport science. In the following sections we report the main findings from studies examining the physiological aspects of soccer referee training and performance.

\section{Match Performance}

\subsection{Distance Covered}

Match analysis and time motion analyses have been extensively used to evaluate the match demands in soccer. ${ }^{[5,11,20-22]}$ Published studies on soccer refereeing match analysis have been available since 1988. ${ }^{[23]}$ During an elite soccer match, referees have been reported to cover an average distance that ranges from 9 to $13 \mathrm{~km}$ (table I).

Asami et al., ${ }^{[23]}$ using hand notational analysis, reported average match coverage of $9990 \pm 927 \mathrm{~m}$ (range 8054-11 180m) in referees officiating in Japanese tournaments. In referees officiating in the Australian soccer first division, Johnston and McNaughton ${ }^{[24]}$ found an average total distance of 9408 $\pm 838 \mathrm{~m}$. Similar results $(9438 \pm 707 \mathrm{~m})$ were reported by Catterall et al. ${ }^{[25]}$ for English Premier League soccer referees. Approximately $2 \mathrm{~km}$ longer distances (11 469 $\pm 983 \mathrm{~m})$ were reported by D'Ottavio and Castagna ${ }^{[26]}$ analysing 96 Italian Serie A matches (36 referees). In this study, large inter-individual differences in total coverage were observed, ${ }^{[26]}$ ranging from 7818 to $14156 \mathrm{~m}$. In Danish top-class soccer referees, Krustrup and Bangsbo ${ }^{[27]}$ calculated average total match coverage of $10070 \pm 130 \mathrm{~m}$ (range 9200-11 490m). Recently, Castagna et al. ${ }^{[28]}$ found in Italian national- and international-level soccer referees total match coverage of $12956 \pm 548$ and $11218 \pm 1056 \mathrm{~m}$, respectively $(\mathrm{p}<0.05)$.

Harley et al. ${ }^{[29]}$ reported for English county-level soccer referees $(n=14)$ an average match coverage of $7496 \pm 1122 \mathrm{~m}(5760-8979 \mathrm{~m})$. This figure represents $65 \%$ and $74 \%$ of the distance reported by D'Ottavio and Castagna ${ }^{[26]}$ and Krustrup and Bangsbo $^{[27]}$ for Italian and Danish elite-level soccer referees, respectively. Consequently, it could be argued that the competitive-level differences in performance reported for soccer players ${ }^{[11]}$ are also evident in referees. However, Castagna et al., ${ }^{[28]}$ comparing elite-level Italian soccer referees (Italian Serie A matches) with international-level referees (European Cups matches), found significantly longer match coverage in the national-level officials (12956 \pm 548 vs $11218 \pm 1056 \mathrm{~m}$ ) [approximately $15 \%$ more 


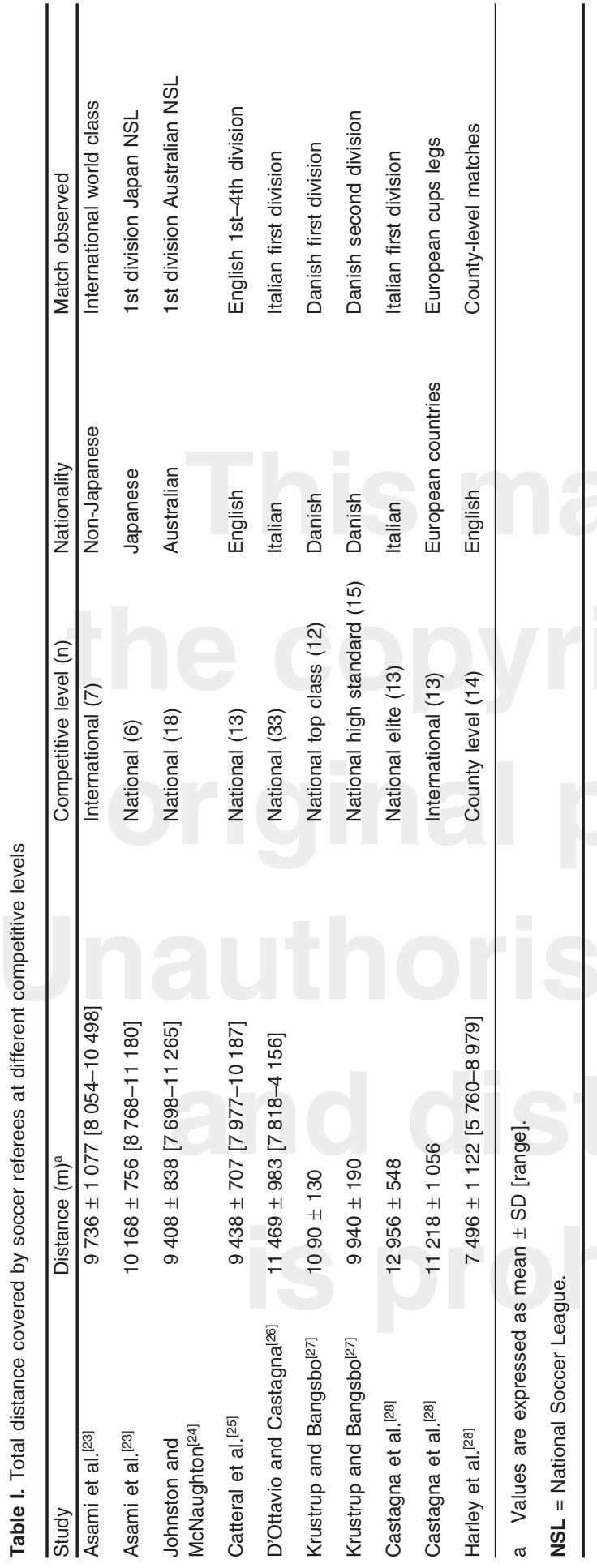

for national level]. In the first study analysing match coverage in soccer referees, Asami et al. ${ }^{[23]}$ found no significant difference in total match distance in FIFA-registered Japanese and foreign referees officiating in domestic and world-class-level matches (10 $168 \pm 756$ and $9736 \pm 1077 \mathrm{~m}$, respectively). Krustrup and Bangsbo, ${ }^{[27]}$ in their study, found no difference in total distance covered by top-class (Danish first division) and high-standard (Danish second division) soccer referees officiating domestic matches.

It could be speculated that the differences observed in match coverage may be related to competitive level when progressing from county-regionallevel competitions to national-international competitions. However, those differences may disappear when dealing with national-international matches. ${ }^{[23,28]}$ Distinct refereeing strategies may account for differences in match coverage at high competitive level. ${ }^{[28]}$ This could be particularly true when referees of different countries are compared. ${ }^{[27,28]}$

Although the participants of the reported studies were all able to pass FIFA and UEFA fitness minimum standards, ${ }^{[16]}$ individual differences in physical fitness level may have affected match performances. ${ }^{[27,28,30-32]}$ Total distance coverage has been reported to represent only a gross variable of match performance in soccer players. ${ }^{[33]}$ However, compared with soccer players, total distance may be considered as a more meaningful variable in referees. In fact, Harley et al. ${ }^{[29]}$ found that total coverage and optimal positioning, considered as the ability to be in the right place at the right time during the match, were significantly related $(\mathrm{r}=0.80 ; \mathrm{p}<$ 0.01). Although related to better positioning, no studies have been carried out to examine possible relationships between match coverage and judgment quality.

The figure of soccer referees' total distance match coverage seems to parallel that reported for midfield players. ${ }^{[19,34]}$ Probably midfield players acting as a link between defence and attack lines and the referee's requirement to keep up with the game are the reason for similarities in match coverage. Aerobic fitness level has been reported ${ }^{[27,31,32]}$ as one 
of the most important factors contributing to space coverage during competition in elite-level soccer referees. However, refereeing strategies ${ }^{[29]}$ and team aggressiveness may also play a role in determining total match coverage.

Collectively, these studies suggest that although differences in match analysis methods and how the activity profiles were categorised may partly account for differences in the total distance covered; the data reported seem to support a trend towards an increase in total distance covered in recent years. These findings are in line with those reported by Williams et al. ${ }^{[35]}$ for soccer players and may be considered as a consequence of increased match demands in soccer in recent years. The total distance covered by referees also appears to be competitivelevel-dependent, especially between national and regional levels of competition.

\subsection{Match Activities}

Although related to better positioning, ${ }^{[29]}$ and considered as a prerequisite for fair judgment, total distance covered does not accurately reflect actual match intensity. In fact, dividing average match total coverage $^{[32]}$ with the average match duration (90 minutes) results in a mean speed of $7.3 \mathrm{~km} / \mathrm{hour}$, a speed much slower than that observed in elite referees running at a blood lactate concentration of 2 $\mathrm{mmol} / \mathrm{L}$ (10.9 km/hour). ${ }^{[32]}$ This is also at odds with the average heart rate (HR) observed during matches, which is usually around 170 beats/min. This anomaly occurs because higher-intensity activities are usually performed over very short periods, yet the physiological response and associated recovery generally last longer than the time required to perform that activity. For example, the average time spent running at high intensity $(\approx 18 \mathrm{~km} /$ hour $)$ is approximately 3 seconds, yet the HR will remain elevated even when the player or referee has decreased their running speed. ${ }^{[26,36,37]}$ With respect to this, better understanding of the physical demands imposed on soccer referees may be acquired by splitting total distance into intensity-related match categories.
Match activities are usually reported as discrete distance or time quantities; however, soccer refereeing is a highly intermittent exercise mode. Timemotion analysis has revealed that elite-level referees change exercise mode approximately every 4 seconds, totalling 1268 activity changes during an average competitive match. ${ }^{[27]}$ Of these 1268 activity changes, 588 and 161 are a consequence of lowintensity and high-intensity running match-activities, respectively. ${ }^{[27]}$

During an average soccer match (90-95 minutes), an elite-level referee has been reported to stand still for about $11-22 \%$ of match duration. ${ }^{[26-28]}$ This may be due to several reasons, such as match intensity, competitive level, fitness level of the referees involved and refereeing strategies. ${ }^{[28]} \mathrm{How}-$ ever, standing is the most easily detectable match variable and enables consistent reasoning about refereeing performance across studies. It could be argued that the differences in standing are the cause of the reported variations in match coverage among studies. In this regard, Castagna et al. ${ }^{[28]}$ reported for international and national referees significant differences in match total coverage with no differences in time spent standing still during the match. Similar results were reported by Krustrup and Bangsbo, ${ }^{[27]}$ who found the same proportions of time spent standing still with no differences in match distance in topclass and high-standard Danish soccer referees. Interestingly, these authors ${ }^{[27]}$ found that high-standard referees stood more during the match compared with their colleagues belonging to the higher competitive standard $(170 \pm 10$ vs $142 \pm 5$ seconds; $\mathrm{p}<0.05)$. This strategy probably enabled highstandard referees to perform more high-intensity activity than the top-class counterpart during the match $^{[27]}$ and demonstrates the partial compensative nature of low-intensity activities in soccer refereeing. In line with this reasoning, Castagna and D'Ottavio $^{[30]}$ showed a strong relationship between fitness level and time spent standing still. In fact, those authors ${ }^{[30]}$ reported a negative correlation $(\mathrm{r}=-0.86$; $\mathrm{p}<0.05)$ between both relative and absolute maximal oxygen uptake $\left(\dot{\mathrm{V}}_{2} \max \right)$ and time spent standing still during the game. An even higher $r$ value $(r=$ 
$-0.90 ; \mathrm{p}<0.05)$ was observed by the same authors ${ }^{[30]}$ when the independent to body mass or scaled $\dot{\mathrm{V}}_{2 \max ^{[38]}}$ was taken into account. In light of this finding, time spent standing still may be considered as a sign of fitness level in soccer refereeing.

During competition, soccer referees have been shown to cover most of the match distance moving at low intensity. ${ }^{[23-25,27,36]}$ These authors considered as low intensity those match activities performed within a 3-13 km/hour speed range. Walking, jogging and low-speed running (6-13 km/hour) are usually considered as low-intensity activities. ${ }^{[18]}$ With this integrated match category, referees have been reported to cover a distance that ranges between $41.8 \%$ and $73.8 \%$ of match coverage. ${ }^{[23,24,26-29]}$ This reveals that a great deal of match time is spent moving at low intensity and if we also add to this the time spent standing still, the figure is even higher. Those researchers addressing the possible effect of fitness status on match performance reported moderate to good $(\mathrm{r}=-0.71$ to -0.81 ; $\mathrm{p}<0.05$ ) correlations between walking (time or distance) and $\dot{\mathrm{VO}}_{2 \text { max }}{ }^{[30]}$ These findings suggest that referees who engage in more low-intensity activities (walking and standing still) during the match probably have low levels of aerobic fitness.

In an attempt to keep up with the tempo of the game, referees perform a considerable amount of activities that are performed at medium and maximal speed. In the available soccer refereeing-related literature, those activities are defined as mediumand high-intensity activities. ${ }^{[18]}$ Again, differences exist in the categorisation of medium- and highintensity activities for the reasons already addressed. However, medium- and high-intensity activities have been defined as those actions performed at speeds that range between $13-18 \mathrm{~km} /$ hour and from $18 \mathrm{~km} /$ hour to maximum speed, respectively. ${ }^{[18]}$ Those speeds are faster than that reported ${ }^{[32]}$ to elicit a $4 \mathrm{mmol} / \mathrm{L}$ blood lactate concentration $(13.6 \mathrm{~km} /$ hour) in elite-level referees, which is widely considered as a critical exercise intensity. ${ }^{[39]}$ Referees have been reported to perform at medium intensity for about $30.2 \%{ }^{[27]}$ of match time or $11-46.3 \%{ }^{[23,25-29]}$ of total distance covered. High-intensity activities account for about $4-18 \%{ }^{[23,26-28]}$ of match distance or $7 \%{ }^{[27]}$ of total match playing time. Sprintingstriding (depending on the authors' categorisation definition), range from $1.7 \%$ to $11.8 \%$ of match total distance. ${ }^{[25-29]}$ Castagna et al. ${ }^{[30,32]}$ and Krustrup and Bangsbo $^{[27]}$ have shown that aerobic fitness plays an important role in determining the distance covered at medium and high intensity. Given that low-intensity activities are negatively correlated with aerobic fitness, ${ }^{[30]}$ and very often used by the referee as a form of active-passive recovery ${ }^{[18]}$ other than for tactical purposes, it could be speculated that highintensity activities heavily affect match coverage in the elite field official.

Although performed at lower speeds, non-orthodox ${ }^{[40]}$ directional modes such as backward and sideward running are physically demanding for the field official. In fact, those directional modes ${ }^{[40]}$ have been shown to add an extra $20 \%$ energy cost compared with speed-matched forward running bouts. Backward running seems to account for a considerable amount of match distance in refereeing as those studies that recorded this match activity reported backward running to range between $6.9 \%$ and $18.2 \%$ of total distance covered. ${ }^{[23-28,36,41]}$ The referees usually adopt backward running in order to take off from the action while still controlling it. However, this directional mode should be limited to a minimum, being physiologically demanding and allowing only slow speeds. ${ }^{[40]}$ Running sideward, the referees cover a limited proportion of match distance (table II) and usually this activity is used as a coordinative link between other directional modes of running, such as backward and forward running.

\subsection{Match-Activities Profile}

A soccer match is played in two halves, each lasting 45 minutes plus allowance for time lost strictly awarded at the referee's discretion. ${ }^{[17]} \mathrm{Be}$ tween halves, 10-15 minutes of rest is allowed to players and officials before the start of the second half. Consequently, the between-halves profile of match activities can be used to examine refereeing strategies and/or the likelihood of acute or progressive fatigue. 


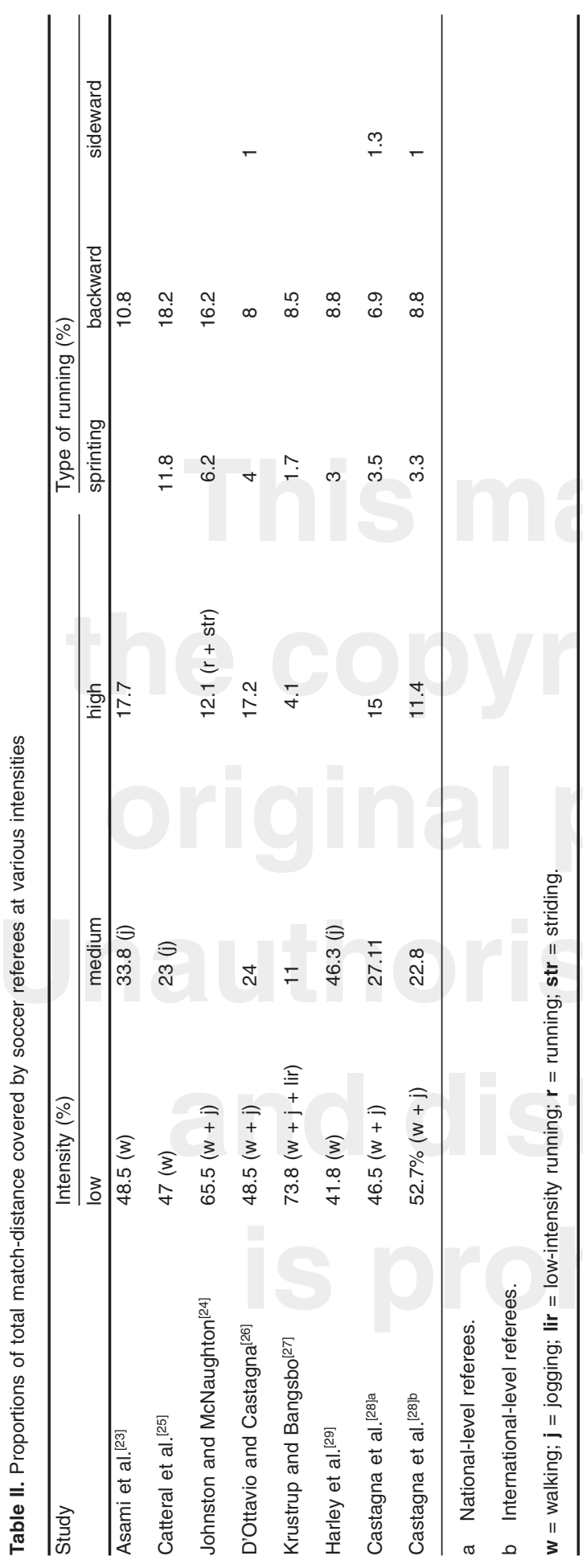

Conflicting results have been reported about first- versus second-half variations in total distance. D'Ottavio and Castagna ${ }^{[26]}$ reported a significant decrement $(-4.1 \%)$ in distance covered during the second half in elite-level Italian soccer referees. Similarly, second-half decrements $(-5.5 \%)$ in distance coverage were found by Catterall et al. ${ }^{[25]}$ for English Premier League soccer referees. Studying the match performance of county-level soccer referees, Harley et al. ${ }^{[29]}$ observed a significant secondhalf decrement of $13.4 \%$. Asami et al. ${ }^{[23]}$ reported in Japanese and foreign referees a $4.7 \%$ second-half increment in total distance covered but the difference was not significant $(p>0.10)$. Johnston and McNaughton ${ }^{[24]}$ and Krustrup and Bangsbo ${ }^{[27]}$ failed to report any half-related variation in total coverage in selected groups of elite Australian high-standard and top-class Danish referees, respectively. More recently, Castagna et al. ${ }^{[28]}$ found no between-halves difference in total distance covered in two groups of Italian elite-level (Serie A) and international-level (UEFA affiliated) soccer referees. In the D'Ottavio and Castagna ${ }^{[26]}$ study, despite a marked decrement in between-halves global coverage, no changes in the high-intensity activity category were observed. Those authors suggested referees adopted a conscious or unconscious sparing behaviour in order to save energy for possible high-intensity activities performed during the latter stages of the match.

High-intensity activity constancy has been confirmed by analysis of selected periods of the match $^{[26]}$ (first and last 15 minutes of each half) and across the games ${ }^{[42]}$ in elite Italian soccer referees. Interestingly, the Italian Serie A referees ${ }^{[26,42]}$ stood still longer during the second half of the match and covered significantly less distance with non-orthodox directional modes in order to maintain the ability to perform at high intensity throughout the game. The documented decrease in the distances covered with backward and sideways running, medium-intensity activity, and a concomitant increase of time standing still demonstrate that the high-level referee develops, through years of officiating; peculiar skills. In fact, referees seem to spare useless activity in order to perform at a high intensity, particularly at 
the end of the match when game intensity often reaches its peak. ${ }^{[2,15,43]}$

Despite the repeated observation of this sparing behaviour among Italian referees, this finding was not confirmed by other authors analysing referees from different countries. Johnston and McNaughton $^{[24]}$ found a significant second-half decrement in high-intensity activities such as running coupled by a significant increment in walking distance during the second half in elite Australian referees. In line with these findings from Johnston and MacNaughton $^{[24]}$ are those more recently reported by Krustrup and Bangsbo ${ }^{[27]}$ in elite-level Danish field referees. In that study, ${ }^{[27]}$ significant decrements in activity performed at high intensity were observed during the second half $(-12.4 \% ; \mathrm{p}<0.05)$. Time-motion analysis revealed that the reported decrement was due to less high-intensity running performed during the first and last 15 minutes of the second half. Differently from the Johnston and MacNaughton ${ }^{[24]}$ study, no variation in low-intensity activities were observed except for a $15 \%$ decrement $(\mathrm{p}<0.05)$ in backward running. In light of the reviewed data, it seems that when elite referees maintain global coverage across halves they experience a significant decrement in high-intensity activities during the second half and particularly during the last periods of play. The size of this decrement in physical performance varies, but is in the range of about $4-5 \%$.

The difference noted between the Italian and Australian-Danish referees seems to confirm the existence of differences in refereeing strategies among elite-level referees. From the physiological and tactical point of view, it would seem advisable for referees to maintain their ability to engage in high-intensity activities across halves in order to successfully produce sprinting and striding activities during the most crucial moments of the game, usually the last 5-15 minutes of the first and second half. ${ }^{[4]}$ This is because refereeing should be regarded as an economy-oriented exercise mode in which the referee, in order to accurately regulate the game, must end the match with the lowest possible mental and physical fatigue. Probably refereeing experience and tactical philosophies are the causes of these differences in high-intensity activities observed between halves. Recently, Castagna et al., ${ }^{[28]}$ comparing Italian and UEFA affiliated referees, found no between-halves difference in high-intensity activities concomitant with no difference in total distance. The referees who were observed in that study were able to cover a total distance lying in the upper range of that usually observed for elite-level referees. As total distance is related to physical fitness, ${ }^{[27,30-32]}$ it could be speculated that aerobic fitness may play a role in the maintenance of across-halves match activity.

\subsection{Work-Rest Ratios}

From the previously reported data, soccer refereeing may be considered as a highly intermittent exercise mode. During an average soccer match, elite-level referees produce a high-intensity effort every 33.5 seconds of play, ${ }^{[27]}$ with each bout lasting approximately 2.3 seconds. Activities performed at low intensity (jogging, low-speed running) are spontaneously undertaken by elite referees over approximately the same duration (2.9 seconds), but more frequently. In fact, in this match category, elite-level referees complete approximately 510 bouts of which $59 \%$ and $41 \%$ are performed with jogging $(8 \mathrm{~km} /$ hour) and low-speed running (12 km/hour), respectively. With low-intensity activities (standing, walking, jogging and low-speed running) elite-level soccer referees have been reported to perform $81 \%$ of the entire match activities. As a result, the high- to low-intensity activity ratio may be defined as $1: 4.3$. This means that, on average, a high-intensity bout occurs every 4-5 low-intensity bouts. Considering match coverage, the high- to low-intensity ratio is $1: 33,1: 6.3,1: 5.8,1: 5.5$ and $1: 6.8$ for English county-level, Danish top-class, high-standard, Italian elite-level, Italian Serie A and international-level soccer referees, respectively. ${ }^{[22,26,29,36,45]}$ The reported high- to low-intensity activity ratio seems to vary remarkably from county level to elite level but to standardise at elite level. The level of fitness and or match intensity seems to play an important role in this, as the referees involved in the Harley et al. ${ }^{[29]}$ study (English county level), al- 
though low skilled compared with the referees competing at elite level, had the same refereeing experience in the respective competitive context. Unfortunately, no detailed information was reported by Harley et al. ${ }^{[29]}$ about county-level referees' physical fitness. However, those referees were able to merely cover $58 \%(7496 \pm 1122 \mathrm{~m})$ of an elite-level referee match distance ${ }^{[28]}$ furthermore, a significant decrement of activity during the second half was evident. The latter finding was explained by Harley et al. ${ }^{[29]}$ as a clear sign of the upcoming fatigue, since the quality of referees' movement patterns deteriorated significantly between the first and second halves.

Match intensity and refereeing strategy may be the cause of the difference reported by Castagna et al. ${ }^{[28]}$ for national and international referees in that comparative study. Krustrup and Bangsbo, ${ }^{[27]}$ comparing top-class with high-standard soccer referees, did not find significant differences with respect to match activities.

\section{Match Physiological Responses}

\section{1 Heart Rate}

Compared with soccer players, HR monitoring is particularly convenient for soccer referees as they are not involved in physical contact during the game. Beside convenience, HR monitoring has shown to also be a fairly reliable match variable in soccer refereeing as intra-individual variation in mean match-HR has been reported to be 4 beats/min with a coefficient of variation of $2 \% \cdot{ }^{[27]}$ Furthermore, the direct validity of HR monitoring has been reported by Helgerud et al. ${ }^{[46]}$ in soccer. In fact, they reported mean match HRs to be related to match performance in soccer players. In soccer refereeing studies, HRs have been reported to be a moderate indicator of match blood lactate concentration ${ }^{[27]}$ and of the aerobic involvement. ${ }^{[37]}$

HR monitoring has been extensively used in soccer refereeing studies with the aim to estimate exercise intensity and aerobic involvement during competition. ${ }^{[24,25,27,36,37,47]}$ These studies have shown that, during competition, an elite-level soccer referee may attain $85-95 \%$ of his maximal $\mathrm{HR}\left(\mathrm{HR}_{\max }\right)$ for the whole match duration. Disparity in the average match HR may be partly due to differences in the way $\mathrm{HR}_{\max }$ has been assessed. In fact, D'Ottavio and Castagna ${ }^{[36]}$ reported for Serie A Italian soccer referees average match HRs being $89 \%$ of the age predicted maximum using the 220 -age formula. Using the same estimation formula, Catterall et al. ${ }^{[25]}$ found in English Premier League soccer referees average match HRs that attained $95 \%$ of maximum. Using the same method, Johnston and McNaughton $^{[24]}$ reported that most of the match time was performed with HRs $>85 \%$ of estimated maximum by Australian top-league soccer referees. Harley et al. ${ }^{[29]}$ found in English county-level soccer referees mean match-HR of $90 \%$ of age-predicted $\mathrm{HR}_{\max }$.

In order to avoid the likelihood of estimation errors, some authors used direct methods in an attempt to calculate the individual $\mathrm{HR}_{\max }$. In this regard, Krustrup and Bangsbo ${ }^{[27]}$ used a progressive treadmill test protocol consisting of stepwise $1 \mathrm{~km} /$ hour speed increments (starting with $14 \mathrm{~km} /$ hour for 2 minutes and then $16 \mathrm{~km} /$ hour for 30 seconds) until exhaustion in order to examine the HR responses to maximal exercise. $\mathrm{HR}_{\max }$ was considered as the peak values reached in 5-second periods at exhaustion. With this direct method, the obtained match mean HR was $85 \%$ of the individual $\mathrm{HR}_{\max }$ for Danish elite-level referees. Weston and Brewer, ${ }^{[47]}$ during English professional soccer matches, found match mean HR of $87 \%$ of HR $\max (153 \pm 7$ beats/ min), which is slightly lower than that reported in other studies. The lower values might be a result of the HR being measured once every 60 seconds, which would tend to reduce the mean HR over long periods.

Helsen and Bultynck ${ }^{[18]}$ found an average match HR of $85 \%$ of $\mathrm{HR}_{\max }$ in UEFA top-class referees during highly competitive matches (2000 European Championship Finals). In contrast to other studies, the Belgian researchers ${ }^{[18]}$ considered for their investigation peak values obtained among different testing protocols, such as laboratory testing, training sessions and match HR analysis as signs of $\mathrm{HR}_{\max }$. They were guided in this methodology by the observation that laboratory testing $\mathrm{HR}_{\max }$ was just $96 \%$ 
and $95 \%$ of match peak and age predicted $\mathrm{HR}_{\max }$, respectively. ${ }^{[18]}$

Those authors ${ }^{[18]}$ suggested that although match and 220-age formula gave similar average HR responses, it did not hold true on an individual basis. These considerations on one hand lend support to the value of individual $\mathrm{HR}_{\max }$ assessment for training prescription, yet on the other hand support the reliability of those findings obtained in referees' studies that adopted the 220-age formula. ${ }^{[24,25,36]}$ Although Helsen and Bultynck ${ }^{[18]}$ found as high as $4 \%$ difference between laboratory and field $\mathrm{HR}_{\max }$ assessment, Krustrup and Bangsbo ${ }^{[27]}$ reported peak match $\mathrm{HR}_{\max }$ as being $97 \%$ of laboratory direct assessment. The reported protocol-dependent difference in $\mathrm{HR}_{\max }$ values may be due to insufficient familiarisation and/or limited motivation of soccer referees during lab testing or the use of unsuitable testing protocols such as cycle-ergometry, as in Helsen and Bultynck ${ }^{[18]}$ no information about the laboratory protocols used is reported. It should also be considered that $\mathrm{HR}_{\max }$ may be affected by training status as some authors reported altered HR $\max$ values in overreached and overtrained athletes. ${ }^{\text {[8-54] }}$ Consequently, biochemical or psychological assessment of training status may prove useful in order to measure the real $\mathrm{HR}_{\max }$ in soccer referees.

Over the duration of a match, D'Ottavio and Castagna ${ }^{[36]}$ reported that Serie A referees exercised at the same HRs across halves, but found significantly less cardiovascular stress during the first 15 minutes of play compared with the two remaining 15-minute sections of the first half. In highly competitive matches, Helsen and Bultynck ${ }^{[18]}$ observed significant variation in HR responses across the game, with the first and the last 15 minutes of each half being the lowest and highest HRs, respectively. Similar across-match 15-minute HR progressions were reported in Danish National Premier League matches, although no between-halves differences (p > 0.05) in mean HR were observed. ${ }^{[27]}$ The across-halves first to last 15 minutes of play HR progression may be regarded as a sign of the increasing physical demands an elite soccer referee is facing during highly competitive matches in an attempt to parallel the tempo of the game. ${ }^{[44]}$ Besides variations in game intensity, other factors such as dehydration, thermal and emotional stress, isometric contractions and high-intensity exercise ${ }^{[19]}$ may help explain these changes in HR. In this regard, those studies that did not find significant between-halves variation in mean $\mathrm{HR}$ a significant decrement in activity was reported, which may have masked the appearance of a half-to-half cardiovascular drift. ${ }^{[25,27]}$ A more detailed analysis of match segments revealed that despite maintenance of highintensity activities across the halves, covering more total distance at the beginning of the match did not result in an increase in mean HR. ${ }^{[36]}$ Similarly, Krustrup and Bangsbo ${ }^{[27]}$ reported a 5 beats/min HR increase across the first half with no significant variations in high-intensity activity. These results may be considered a consequence of the occurrence of a progressive dehydration or increasing thermal stress, conditions that probably were exacerbated during the second half where a progressive increment of average 15-minute HRs was paralleled by a progressive decrement of high demanding activities. ${ }^{[27]}$

\subsection{Aerobic Involvement}

During an average competitive first-division match, a referee works for $53-56 \%$ and $28-33 \%$ of the match time at HRs between 70-90\% and $>90 \%$ of the individual $\mathrm{HR}_{\max }$, respectively. ${ }^{[27,55]}$ This clearly shows that aerobic metabolism is heavily taxed during competition.

Although match HR monitoring has limitations in the estimation of game aerobic involvement, the proved convenience and feasibility has promoted its use. ${ }^{[4,19,56]}$ In fact, several researchers have attempted to estimate match oxygen uptake $\left(\dot{\mathrm{VO}}_{2}\right)$ and thus aerobic involvement by taking advantage of the linearity of the $\mathrm{HR}-\dot{\mathrm{V}} \mathrm{O}_{2}$ relationship established during progressive treadmill running to exhaustion. ${ }^{[57]}$ The estimated aerobic involvement during competitive games has been found to be about $81 \%$ of the $\dot{\mathrm{V}} \mathrm{O}_{2 \max }$ assessed by a treadmill running test. No differences seem to be detectable across different competitive levels as Krustrup and Bangsbo ${ }^{[27]}$ 
and Weston and Brewer ${ }^{[47]}$ reported an estimated $\dot{\mathrm{VO}}_{2 \text { max }}$ in elite-level soccer referees of $80.5-81 \%$ and Harley et al. ${ }^{[29]}$ calculated a mean intensity of $80 \%$ of $\dot{\mathrm{VO}}_{2 \text { max }}$ for county-level officials of similar age. Although significant differences in match activities during the second half were reported by Harley et al. ${ }^{[29]}$ as a sign of progressive fatigue, they did not observe any significant difference in the estimated match $\dot{\mathrm{V}} \mathrm{O}_{2 \text { max }}$ proportion across halves $(81 \%$ and $79 \%$ of $\dot{\mathrm{VO}}_{2 \max }$, for the first and second half, respectively; $p>0.05)$. Data regarding referees competing at elite level have shown conflicting results. In fact, significantly less aerobic involvement $(-2.5 \% ; \mathrm{p}<0.05)$ during the course of the second period of play has been reported by Weston and Brewer $^{[47]}$ in Premier League referees. However, no between-halves differences were found by Krustrup and Bangsbo ${ }^{[27]}$ in Danish top-class referees. This was despite that during the second half a significant decrement in the most demanding match activities such as high-intensity and backward running was reported. The difference observed in the proportion of $\dot{\mathrm{VO}}_{2 \max }$ across halves is probably due more to bias in HR behaviour rather than real $\dot{\mathrm{VO}}_{2}$ drift.

These values are higher than those reported for soccer players in studies that used similar testing protocols and the $\mathrm{HR}-\dot{\mathrm{V}} \mathrm{O}_{2}$ relationship to estimate the match aerobic involvement. ${ }^{[2,5,19]}$ In those studies, soccer players have been reported to work during competitive matches at an estimated exercise intensity of $70-75 \%$ of $\dot{\mathrm{VO}}_{2 \text { max }}$. The resulting $7-14 \%$ higher aerobic demand in soccer refereeing may be as a consequence of the referee's requirement to keep up with the game, whatever the tempo is, in the attempt to be at the right place at the right time. In fact, players have potentially a higher degree of choice in undertaking high-intensity efforts off 'the ball' compared with the referee.

D'Ottavio and Castagna, ${ }^{[37]}$ in an attempt to spread light on the real aerobic demand of soccer refereeing, directly measured in elite-level soccer referees match $\dot{\mathrm{VO}}_{2}$ responses using a portable lightweight gas analyser (K2, COSMED, Rome, Italy). During this study, referees officiated friendly matches with the help of a competitive-level matched colleague that whistled for them when they called for an infringement by raising an arm. With the aim to control for a possible detrimental effect on match work-rate caused by the portable gasanalyser, match analysis was performed with a computerised system. ${ }^{[26]}$ Results showed that over the observed match period (45 minutes, first half) the referees attained $68 \%$ of the individual $\dot{\mathrm{VO}}_{2 \max }$. Match coverage $(6614 \mathrm{~m})$ and mean match HR $(89 \%$ of individual $\mathrm{HR}_{\max }$ ) were both in line with that observed in age and competitive-level matched referees during Italian Championship official matches. ${ }^{[26,28,36,58]}$ The use of the treadmill HR- $\dot{\mathrm{V} O} 2$ relationship resulted in a significant $(\mathrm{p}<0.001) 9 \%$ overestimation of the actual match- $\dot{\mathrm{VO}}_{2}$, referees attaining $74.3 \%$ of $\dot{\mathrm{V}}_{2 \text { max }}{ }^{[37]}$ The direct analysis of aerobic demands clearly show that soccer refereeing taxes the aerobic system to a level that is lower than that reported in those studies that estimated the match- $\dot{\mathrm{V}} \mathrm{O}_{2}$ through the maximal progressive protocol HR- $\dot{\mathrm{V}}_{2 \max }$ relationship.

Using the same portable gas analysis system, Rodriguez and Iglesias ${ }^{[59]}$ found match $\mathrm{VO}_{2}$ values that ranged from $43 \%$ to $69 \%$ of $\dot{\mathrm{VO}}_{2 \text { max }}$ in professional soccer players during friendly matches. The reported values are within the $\mathrm{V}_{2}$ range found by D'Ottavio and Castagna, ${ }^{[37]}$ but quite lower than those estimated in soccer players using the laboratory $\mathrm{HR}-\dot{\mathrm{V}} \mathrm{O}_{2}$ relationship. ${ }^{[2,5,19]}$ In an attempt to examine the real importance of match-HR, D'Ottavio and Castagna ${ }^{[37]}$ calculated match $\mathrm{HR}-\mathrm{VO}_{2}$ regression lines finding moderate correlations (0.68-0.70; $\mathrm{p}<0.001)$. These values are quite lower than those usually found during laboratory treadmill testing for $\dot{\mathrm{VO}}_{2 \text { max }}{ }^{[57]}$ Consequently, the use of lab HR- ${ }^{5} \mathrm{O}_{2}$ regression lines should be made with caution when attempting to outline the aerobic involvement during soccer refereeing.

Data available on the aerobic demands of soccer refereeing seem to confirm that the aerobic system is heavily taxed during the match, although caution should be paid when interpreting the results obtained using the laboratory $\mathrm{HR}-\dot{\mathrm{V}} \mathrm{O}_{2}$ relationship as an estimating tool. 


\subsection{Blood Lactate}

Blood lactate concentrations measured during exercise provide a gross indication of the involvement of anaerobic glycogenolysis. While the intermittent activity profile of the soccer referee suggests the involvement of both aerobic and anaerobic energy systems, it is of interest for the development of training programmes to examine the extent to which anaerobic glycogenolysis is activated in the soccer referee during competitive matches.

Compared with soccer player-related scientific literature, ${ }^{[5,19,44]}$ only two papers have reported data on match blood lactate concentrations in soccer referees. ${ }^{[27,37]}$ Furthermore, only one study used blood samples drawn during the game. ${ }^{[37]}$ In Danish top-class $^{[27]}$ and high-standard referees, blood lactate concentrations of 4.8 and $5.1 \mathrm{mmol} / \mathrm{L}$ have been reported at the end of the first and second half, respectively. These values are similar to those previously reported in soccer players of the same competitive level in studies that used the same blood sample timing. ${ }^{[5]}$ Blood sample timing has been reported to affect the resulting values of lactate concentration in soccer players. ${ }^{[20]}$ In fact, Smith et al. ${ }^{[60]}$ showed large differences in blood lactate concentrations between post-halves and random sampling protocols during friendly games.

Large inter- and intra-individual differences in blood lactate concentration seem to exist in elitelevel referees. ${ }^{[27]}$ This high variability in blood lactate concentrations is probably due to the activity intensity before blood sampling. In this regard, Krustrup and Bangsbo ${ }^{[27]}$ and Bangsbo ${ }^{[20]}$ reported significant correlations between activity intensity attained before blood sampling and the resulting lactate concentration in competitive-matched referees and soccer players, respectively. Blood lactate concentrations as high as $14 \mathrm{mmol} / \mathrm{L}$ have been reported at the end of the second half that are significantly higher than the corresponding peak value measured at the end of the first half. ${ }^{[27]}$ These findings clearly show that elite-level soccer referees experience high lactate concentrations in crucial moments of the game. D'Ottavio and Castagna, ${ }^{[37]}$ studying match blood lactate profiles in Serie A soccer referees officiating friendly matches, found first-half blood lactate concentration slightly $>7$ $\mathrm{mmol} / \mathrm{L}$ using during-competition blood sampling. In that study, game blood lactate concentrations were significantly higher than the post-warm-up level $(2.1 \pm 0.5 \mathrm{mmol} / \mathrm{L})$. The high intra-individual variability (coefficient of variation $=31 \%$ ), the documented game-intensity dependency and the high blood lactate concentration sometimes found seem to suggest that while 'lactate tolerance' may be important for the referee to develop, the measurement of blood lactate during a match is not a reliable indicator of referee performance or fitness. However, the lack of detailed data on the issue of blood lactate production during the game warrants further research.

\subsection{Hydration Status}

Performance ability has been reported to be strongly linked to hydration status. ${ }^{[57]}$ It has been shown that fluid losses totalling only $2 \%$ of body mass can induce an impairment of aerobic performance by as much as $20 \% .{ }^{[61]}$ Although performance decrements are evident even with a fluid deficit exceeding about $1 \%$ of body mass ${ }^{[62]}$ in the presence of a cold $\left(5-10^{\circ} \mathrm{C}\right)$ or temperate $\left(21-22^{\circ} \mathrm{C}\right)$ environment, $2 \%$ of body mass fluid loss may be easily tolerated by well trained individuals. ${ }^{[63]}$ Fluid loss is exacerbated by environmental conditions such as heat, high humidity, lack of wind and/or fluid restriction ${ }^{[62]}$ and by high-intensity activities that require dissipation of more metabolic heat. ${ }^{[61,64]}$ It is generally accepted that cognitive performance is also impaired when dehydration and hyperthermia are present. ${ }^{[65]}$

During competitive soccer matches, large fluid losses, even in a relatively cold environment, have been reported in elite soccer players. Davies et al. ${ }^{[66]}$ found in Premier League professional soccer players as much as $2.9 \%$ of weight loss competing in environmental temperatures of $7^{\circ} \mathrm{C}$ and $81 \%$ relative humidity. During World Cup qualifying matches played in hot conditions $\left(30^{\circ} \mathrm{C}, 40 \%\right.$ relative humidity), Mustafa and Mahmoud ${ }^{[67]}$ observed an average $3 \%$ body mass decrement due to evaporative fluid 
loss in acclimated elite soccer players. Fluid loss was similar in colder conditions $\left(26.3^{\circ} \mathrm{C}\right)$ with higher relative humidity (78\%). ${ }^{[67]}$ Ekblom $^{[11]}$ observed in elite soccer players competing at temperatures of $\geq 30^{\circ} \mathrm{C}$ a decrement of $50 \%$ in the distance performed at high intensity compared with a control condition $\left(20^{\circ} \mathrm{C}\right)$, with the reduction being especially evident in the second half. Environmental temperature did not affect total distance covered, showing that players probably change playing strategies when in hot conditions. ${ }^{[11]}$ McGregor et al., ${ }^{[68]}$ using an intermittent shuttle test designed to simulate the performance demands of soccer, ${ }^{[69]}$ found deteriorated sprint $(15 \mathrm{~m})$ performances in semi-professional soccer players during the last 15 minutes of the 90-minute simulation protocol when no fluid was ingested. This condition elicited a body mass decrement of $2.4 \%$ exercising in a cold to temperate environment $\left(13-20^{\circ} \mathrm{C}, 57 \%\right.$ relative humidity). The $1.4 \%$ body mass decrement observed during the water ingestion condition $(2 \mathrm{~mL} / \mathrm{kg}$ every $15 \mathrm{~min}$ utes) did not impair sprint performance throughout the six 15-minute periods of the intermittent shuttle test.

According to the available literature on dehydration in soccer, it is clear that fluid losses corresponding to $2 \%$ of bodyweight are easily achieved by players whatever the environmental condition and provided that proper re-hydration is not considered. Furthermore, significant physical and skill ${ }^{[68]}$ performance decrements are expected as a consequence of dehydration in soccer players.

To date, limited research has examined the issue of fluid loss in soccer referees. Da Silva and Fernan$\mathrm{dez}^{[70]}$ observed a $2 \%$ fluid evaporation-induced bodyweight loss after a competitive match in elite Brazilian soccer referees. This was observed in environmental conditions of $20.3^{\circ} \mathrm{C}$ and $76.8 \%$ air temperature and relative humidity, respectively. Slightly greater bodyweight decrements (2.5\%) were reported by Krustrup and Bangsbo ${ }^{[27]}$ for Danish elite-level soccer referees, during domestic matches played in autumn to winter. These findings are similar to those previously reported for soccer players. It could be speculated that referees may experience the same level of dehydration found in soccer players during competition in comparable environmental conditions. However, referees are expected to follow the game quite closely from the beginning to the end without any down time. ${ }^{[36]}$ This is often accomplished irrespective of the intensity of previous activities. ${ }^{[36]}$ Given that, higher levels of dehydration may be expected in referees compared with players competing during the same match.

Additionally, the level of dehydration found in soccer referees are of the same magnitude of those reported to have detrimental effects on physical ${ }^{[61,62]}$ and cognitive ${ }^{[65]}$ performance. This has potentially negative effects on referee judgments and particularly so during the last stage of a competition, where the urgency of the game has been reported to increase $^{[15,44,71,72]}$ and the dehydration is greater. ${ }^{[11]}$ Probably fluid loss can be considered as one of the causes that determined the significantly longer distance from infringements observed in the last 15 minutes of play and the shorter distance covered at high intensity during the second half in dehydrated referees as reported by Krustrup and Bangsbo. ${ }^{[27]}$

As FIFA's rules do not allow fluid replacement during the game, referees should necessarily hydrate during match intervals as far as possible. However, Da Silva and Fernandez ${ }^{[70]}$ reported that only $24 \%$ of fluid loss was replaced by referees with ad libitum fluid ingestion during match intervals. In order to optimise referee physical and cognitive performance throughout the game, the use of re-hydration fluid should be systematic and accurately scheduled during competition and the laissez faire attitude discouraged. ${ }^{[66]}$ Particular attention should be paid when playing in hot conditions $\left(>30^{\circ} \mathrm{C}\right)$ as a dehydration by $2 \%$ of body mass impairs absolute power production and predisposes individuals to heat injury. ${ }^{[63]}$

Although dehydration has been reported to negatively affect cognitive performance, ${ }^{[65,73]}$ the results should be applied with caution to soccer refereeing as no specific study has been carried out so far. McGregor et al. ${ }^{[68]}$ found no deterioration in number-individuation performance after a soccer-specific protocol lasting 90 minutes as a consequence of 
dehydration (2.4\% body mass loss). However, the difference in cognitive abilities requested during soccer refereeing ${ }^{[18]}$ compared with number-individuation tests are evident. As the decision-making process is considered the most important aspect of refereeing performance, referees may gain an advantage from ingesting carbohydrate-enriched drinks as Reilly and Lewis ${ }^{[73]}$ reported that carbohydrate feeding was superior to simple water ingestion in maintaining decision-making during prolonged exercise.

These results clearly show that, like soccer players, referees may experience significant dehydration as a consequence of officiating. Water ingestion during intermittent exercise has been shown to have a beneficial effect on physical performance. ${ }^{[68]}$ Consequently, fluid ingestion should be promoted during soccer refereeing and particularly during extreme environmental conditions that are likely to occur in hot seasons and in tropical and subtropical countries. Association football international governing bodies (FIFA and UEFA) should consider rules modifications in order to allow fluid replacement during tournaments played in extreme environments such as World Cups and European Championships.

The thermal strain on the individual referees is a function of the relative exercise intensity (percentage of $\dot{\mathrm{VO}}_{2 \max }$ ) rather than the absolute work load. ${ }^{[74}$ Therefore, aerobic power should be improved in soccer referees in order to lower the thermal stress during competition. In addition to proper drinking and fitness training, the negative effect of dehydration may be limited with proper acclimation. ${ }^{[44]}$

\section{Physical Capacities of Soccer Referees}

\subsection{Anthropometry}

The age of the referees who officiated in the more recent World Cup and UEFA Cup finals are on average $15-20$ years older ${ }^{[18]}$ than the professional players whose behaviour they have to regulate. ${ }^{[19]}$ Furthermore, the referees' age reported in the inter- national literature clearly shows that elite soccer referees may reach the top of their officiating careers at ages that are even older than 40 years. ${ }^{[18]}$ Rontoyannis et al. ${ }^{[75]}$ found in a group of Greek referees representing the first four top soccer divisions of Greece, that first-division referees were 3-4 years older than the lower-competitive-level officials. However, even at lower competitive levels, ${ }^{[29,41,75,76]}$ referees have average ages that easily exceed 35 years. This older than expected age, even at lower competitive levels, has been explained by Harley et al. ${ }^{[29]}$ as a consequence of previous participation in active soccer before becoming county-level referees. In contrast to players, a soccer referee's career progression is strongly related to age. In fact, talented soccer referees progress from one competitive level to the next with no possibility of skipping a level based on officiating skill. ${ }^{[28]}$

Given the mature age usually shown by referees competing at elite level in their respective divisions, it can be assumed that experience is a fundamental pre-requisite for successful refereeing. Nevertheless, international ${ }^{[77]}$ and some national ${ }^{[28]}$ referee governing bodies set a cut-off age of 45 years for soccer officials. However, referees $>45$ years of age have been reported to be still active in Denmark (47 years) ${ }^{[27]}$ and Greece (46 years). ${ }^{[75]}$

Body mass index (BMI) is usually reported as a rough measure of body composition ${ }^{[78]}$ and a good correlate of relative body fat. Helsen and Bultynck $^{[18]}$ reported BMI values of $24.2 \pm 2.6 \mathrm{~kg} /$ $\mathrm{m}^{2}$ in top-class referees participating in the Euro 2000 Championship finals. Higher BMI values were found by Rontoyannis et al. ${ }^{[75]}$ in a mixed group of Greek referees $(n=188)$ whose average value was $25.9 \pm 2.1 \mathrm{~kg} / \mathrm{m}^{2}$. In that study, ${ }^{[75]}$ first-division referees had a BMI of $26.5 \pm 1.1 \mathrm{~kg} / \mathrm{m}^{2}$ and no significant differences were observed between competitive levels. Interestingly, Rontoyannis et al. ${ }^{[75]}$ found $70 \%$ of the studied referees $(n=188)$ were overweight (BMI $\left.>25 \mathrm{~kg} / \mathrm{m}^{2}\right)$ and $6 \%$ were obese $\left(\right.$ BMI $\left.>30 \mathrm{~kg} / \mathrm{m}^{2}\right)$. It appears that only top-class referees possess favourable BMI indices considered as values $<25 \mathrm{~kg} / \mathrm{m}^{2} \cdot{ }^{[75,77,78]}$ However, even in top- 
class referees, BMI values of $31.4 \mathrm{~kg} / \mathrm{m}^{2[18]}$ have been reported.

These results suggest that more attention should be paid by referee governing bodies when selecting officials and particularly when organising international competitions. Proper training and nutrition should be implemented to referees in order to promote optimal performances and health. ${ }^{[16]}$ Furthermore, the documented persistence of elite-level referees between the 35- to 40-year age-range and their prevalent amateur/semi-professional status, raises questions about the general health of this athletic population. ${ }^{[75]}$ Furthermore, as fitness is strongly related to age progression, particular attention should be paid to participation fitness standards. ${ }^{[79]}$

\subsection{Maximal Oxygen Uptake}

Aerobic metabolism is heavily taxed during competitive soccer refereeing, whatever the competitive level is. Additionally, $\dot{\mathrm{VO}}_{2 \max }$ has been reported to affect match coverage. ${ }^{[27,30]}$ Given that, high $\dot{\mathrm{V}} \mathrm{O}_{2 \mathrm{max}}$ levels should be expected in soccer referees. Despite these expectations, the existing literature seems to suggest a moderate $\dot{\mathrm{V}}_{2}$ max, even for the elite referee. Castagna and D' Ottavio ${ }^{[30]}$ reported relative $\dot{\mathrm{VO}}_{2 \max }$ values of $49.30 \pm 8.0 \mathrm{~mL} / \mathrm{kg} / \mathrm{min}$ in elite-level Italian referees. Similar values were reported by Krustrup and Bangsbo ${ }^{[27]}$ for ten Danish top-class referees with their average $\dot{\mathrm{V}} \mathrm{O}_{2 \text { max }}$ being $46.3 \mathrm{~mL} / \mathrm{kg} / \mathrm{min}$. Interestingly, values as low as 40.9 $\mathrm{mL} / \mathrm{kg} / \mathrm{min}$ and peak values not exceeding $56 \mathrm{~mL} /$ $\mathrm{kg} / \mathrm{min}$ were reported. ${ }^{[27]}$ In a larger sample $(\mathrm{n}=27)$ of Danish top-class referees, Bangsbo et al. ${ }^{[80]}$ found $\dot{\mathrm{VO}}_{2 \max }$ levels of $47.7 \pm 1.5,45.9 \pm 1.1$ and $44.7 \pm$ $0.8 \mathrm{~mL} / \mathrm{kg} / \mathrm{min}$ in younger (age 29-34 years), intermediate (age 35-39 years) and older (age 40-46 years) referees, respectively. Weston and Brewer ${ }^{[47]}$ found in English Premier League referees values slightly higher than those reported for Italian and Danish elite-level referees, $\dot{\mathrm{V}} \mathrm{O}_{2}$ max averaging 50.9 $\pm 5.7 \mathrm{~mL} / \mathrm{kg} / \mathrm{min}$. The reported maximal aerobic power values show that irrespective of competitive level, referees possess quite low $\dot{\mathrm{VO}}_{2 \max }$ values when compared with those found in soccer play- ers. ${ }^{[5]}$ In this regard, it seems that success in soccer refereeing may be achieved with $\dot{\mathrm{V}}_{2}$ max levels significantly lower than $50 \mathrm{~mL} / \mathrm{kg} / \mathrm{min}$, a value that may be easily found in moderately active or sedentary people. ${ }^{[78]}$ This figure is quite different from the minimum $\dot{\mathrm{VO}}_{2}$ max threshold suggested by Reilly et al. ${ }^{[81]}$ for achieving performance success in soccer and individualised as being $60 \mathrm{~mL} / \mathrm{kg} / \mathrm{min}$. The finding that improvement in match physical performance may be achieved without significant improvements in $\dot{\mathrm{VO}}_{2 \text { max }^{[27]}}$ suggests that elite-level soccer referees may take advantage of other physiological variables during competition.

\subsection{Anaerobic Performance}

Involvement of the anaerobic pathway, although quantitatively minor, nevertheless plays an important role as high-intensity bouts of activity are particularly important at given moments of the match. ${ }^{[26]}$ During competition, referees are involved in sprinting-striding activities in an attempt to keep up with the game. However, not being involved directly in the game, referees may limit sprinting and high-intensity anaerobic efforts with a well developed sense of position. ${ }^{[29]}$ Sprint bout duration is reported to range between $2-4$ seconds with distance rarely exceeding $30 \mathrm{~m} .{ }^{[26]}$ During a competitive match, referees perform 12-16 sprint-bouts of a reported average duration of 1.7-1.9 seconds, ${ }^{[27]}$ which is quite low compared with players.

The available international literature has only rarely addressed anaerobic performance in soccer referees. Furthermore, in the available studies, no laboratory testing was performed. Castagna et al. ${ }^{[31]}$ tested anaerobic performance of elite-level soccer referees with 50 and $200 \mathrm{~m}$ sprinting reporting average values of $7.07 \pm 0.26$ and $29.57 \pm 1.44$ seconds, respectively. $200 \mathrm{~m}$ performances similar to those reported by Castagna et al. ${ }^{[31]}$ were reported by Barbanti and Bergson ${ }^{[76]}$ for a group of state-level Brazilian referees ( $29 \pm 1.7$ seconds). Rontoyannis et al. ${ }^{[75]}$ found average $200 \mathrm{~m}$ performance times of $31.2 \pm 1.7$ seconds in a mixed group of Greek soccer referees, first-division referees being faster than their third-division colleagues $(30.2 \pm 1.1$ vs $31.5 \pm$ 
1.6 seconds; $\mathrm{p}<0.01)$. Third-division referees ${ }^{[75]}$ were also slower over $50 \mathrm{~m}$ compared with their first-division counterparts $(7.5 \pm 0.3$ vs $7.3 \pm 0.3$ seconds; $\mathrm{p}<0.01)$.

Vertical jump performance is usually used as a valid measure of explosive power in soccer players. ${ }^{[5,38,82]}$ In the only study that addressed vertical jump performance in elite soccer referees, jumping heights of $34 \pm 3 \mathrm{~cm}$ were reported. ${ }^{[79]}$ Interestingly, younger (age 31-35 years) referees showed better jumping performance than average (age 36-39 years) and older (age 40-45 years) officials. ${ }^{[79]}$ To the author's knowledge, no research has been published on the strength performance of soccer referees. This would be of importance for the development of proper training strategies as the age-related decrease in muscular strength has previously been documented. ${ }^{[83]}$

\subsection{Fitness Testing}

International governing bodies (FIFA and UEFA) usually test their affiliated internationallevel soccer referees with field tests such as 50 and $200 \mathrm{~m}$ sprints and the 12-minute running test for distance. ${ }^{[77]}$ Although widely used and currently in use by national referee associations for the assessment of physical fitness of elite and non-elite referees, ${ }^{[31,77]}$ sport scientists have recently proposed new field tests with the aim to add relevance to match performance. In this direction, proposals have been particularly prolific for the aerobic-endurance side of fitness assessment. ${ }^{[10]}$ Although the 12-minute run test has been shown to be related to match physical performance, ${ }^{[32]}$ it involves a running mode (track running) quite different from what is usually observed during soccer refereeing. Moreover, the 12-minute run test requires pace judgement.

Harley et al. ${ }^{[41]}$ proposed a multistage fitness test (Football Referees [FR] test) for the assessment of aerobic fitness that involved activities such as walking, sprinting and jogging that are usually performed during competition by referees. The test protocol was based upon published match analysis data ${ }^{[24,25]}$ and consisted of moving around a square of $50 \mathrm{~m}$ sides at progressive speed following audio instruc- tions from a cassette player. The $100 \mathrm{~m}$ sequence involved $50 \mathrm{~m}$ jogging followed by 6 seconds of passive recovery, a jog backwards for $20 \mathrm{~m}$, turn and sprint for $10 \mathrm{~m}$ and walk for $20 \mathrm{~m}$. The test consists of nine levels of 3 minutes each, with time to complete the $100 \mathrm{~m}$ sequences reduced at the end of each level until exhaustion. The test showed good correlations with the multistage fitness test ${ }^{[84,85]}$ and treadmill $\dot{\mathrm{V}} \mathrm{O}_{2 \max }(\mathrm{r}$ values 0.73 and 0.82 , respectively; $\mathrm{p}<0.001)$. Harley et al. ${ }^{[41]}$ reported subject satisfaction with the new test, because of a perception of task specificity. Those authors ${ }^{[41]}$ reported that FR test data were used to provide training guidance to county-level soccer referees and used by the County League in judging whether an individual referee's fitness is of a suitable level to be eligible for officiating county-level matches. Furthermore, the FR test showed good test retest reliability. ${ }^{[41]}$ Although the FR test may be considered a valuable attempt to devise a soccer refereeing ecological test, the FR test has not yet been tested for direct validity (i.e. against match physical performance).

Recently, Krustrup and Bangsbo ${ }^{[27]}$ proposed the Yo-Yo Intermittent Recovery Test (YYIRT). YYIRT consists of repeated shuttle running over a distance of $20 \mathrm{~m}$ at increasing speed until exhaustion. Every $2 \times 20 \mathrm{~m}$ bout of shuttle running the referee is allowed 10 seconds of active recovery by jogging back and forward over a $5 \mathrm{~m}$ distance. The practical advantages of YYIRT lies in that it allows mass testing using intermittent running (logical validity) with no pace judgement as the speed is dictated by recoded bleeps. However, the strength of this test is in its external validity as it has been shown to be related to match physical performance of elitelevel soccer referees. ${ }^{[27]}$ Specifically, YYIRT revealed a good correlation $(r=0.75 ; \mathrm{p}<0.05)$ with distance covered at high intensity (speed faster than $15 \mathrm{~km} /$ hour), which is considered as a potentially limiting factor in soccer refereeing ${ }^{[26-28,42]}$ and in soccer. ${ }^{[9,20,21]}$ YYIRT was also found to be significantly related to the total distance covered $(r=0.66$; $\mathrm{p}<0.05)$. In contrast to that found for the general population, ${ }^{[86]}$ the YYIRT test was not reported to have a significant relationship with $\dot{\mathrm{VO}}_{2}$ max , show- 
ing that YYIRT may be considered as a specific soccer refereeing test. Recently, the YYIRT has been reported to possess construct validity ${ }^{[58]}$ and training sensitivity ${ }^{[27]}$ in elite soccer referees. Like other field tests, ${ }^{[10]}$ YYIRT may be regarded as a terminal test as test results may not be consistently used to implement training strategies. In this regard, laboratory testing should be preferentially considered when precise measures for fitness development are needed. ${ }^{[10]}$

The use of 50 and $200 \mathrm{~m}$ sprint performance is prone to some criticism also. ${ }^{[31]}$ In fact, no relationship is evident between 50 and $200 \mathrm{~m}$ performances and match performances. ${ }^{[31]}$ Furthermore, the reported testing performance did not mimic what match analysis has reported to take place during the game. ${ }^{[26,27]}$ In this regard, as far as sprint ability is concerned, $30 \mathrm{~m}$ may be considered as a more related to actual performance than $50 \mathrm{~m}^{[26,27]}$ and a repeated sprint ability test $(7 \times 30 \mathrm{~m}$ with 20 seconds of recovery between bouts) instead of $200 \mathrm{~m}$ track sprinting may be more appropriate. ${ }^{[81]}$ These tests are used extensively by sport scientists for soccer players $^{[81]}$ and should also be considered for their applicability to the assessment of elite soccer referees. That being said, there are still very limited data available on the relationships between sprint performance during a match and tests of sprinting ability, such as repeated sprint ability tests.

Physical performance has been reported to deteriorate with aging and recently Castagna et al. ${ }^{[79]}$ showed that the age span in which elite referees are included is likely to experience such performance impairment. Castagna et al. ${ }^{[79]}$ suggested that national and international governing bodies should consider an age-related cut-off performance level instead of the current fixed criteria. ${ }^{[16]}$ In this regard, a 30-year-old elite-level soccer referee should be able to cover at least $3030 \mathrm{~m}$ in the 12 -minute running test in order to pass the $2700 \mathrm{~m}$ cut-off criteria at 42 years, which is approximately the average age of the officials involved in the last stages of the 2002 FIFA World Cup. ${ }^{[58]}$

\section{Training}

Given the evidence presented so far, soccer refereeing may be regarded as a predominantly aerobic activity. Consequently, training strategies should be mainly devoted to improving aerobic metabolism in order to improve the soccer referee's match performance. Unfortunately, only limited literature is currently available on the effect of structured aerobic training sessions on refereeing match performance or physiological parameters per se.

Recently, Helgerud et al. ${ }^{[46]}$ have shown that long interval running sessions $(4 \times 4$ minutes at $90-95 \%$ $\mathrm{HR}_{\max }$ with 3 minutes of active recovery) are effective in enhancing aerobic fitness ${ }^{[87]}$ in youth soccer players. Interestingly, improvements in aerobic power not only enhanced players physical performance (total distance covered), but also enhanced selected match activities such as the number of ball involvements.

Krustrup and Bangsbo ${ }^{[27]}$ using interval running training sessions that included a progressive use of short (30-second and 1-minute) and long (4- and 8-minute) intervals (work-rest ratio $2: 1$ ) for 3-4 days a week for 12 weeks, did not find any significant improvement in $\dot{\mathrm{V}}_{2}$ max and $\dot{\mathrm{VO}}_{2}$ at sub-maximal treadmill speeds (10-16 km/hour) in Danish top-class referees. These results are surprising, as in this study the intensity and the training loads were similar to those reported by Helgerud et al. ${ }^{[46]}$ In fact, Krustrup and Bangsbo ${ }^{[27]}$ used exercise intensities that were for all intervals performed above $90 \%$ of $\mathrm{HR}_{\max }$. Although no improvements of aerobic fitness ${ }^{[87]}$ were observed, the Danish top-class referees reported remarkable improvements in YYIRT $(+31 \%)$ and in the time to exhaustion during a treadmill test $(+7 \%)$. Significant improvements were observed in selected match activities, such as high-intensity running (+23\%) and the average distance from infringements. Improvements in match activities were particularly evident during the second half.

Weston et al. ${ }^{[55]}$ found large improvements in YYIRT $(+46.5 \%)$ in elite Belgian referees after 16 months of specific training. Specific training consisted of track and pitch sessions that elicited HR of 
$88 \%$ and $86 \%$ of $\mathrm{HR}_{\max }$, respectively ( $\mathrm{p}>0.05$ ). High-intensity sessions were undertaken two times per week, with other sessions being recovery and pre-match training sessions (four in all). In that study, pitch sessions were shorter than track sessions ( 20 vs 27 minutes; $p<0.05$ ). In the Weston et al. ${ }^{[55]}$ study, referees who possessed a higher level of fitness at the start of the investigation (i.e. greater distance covered during the YYIRT) showed less aerobic fitness improvements compared with their lower fit counterparts (international vs national referees, $35.5 \%$ vs $53.9 \%$, respectively). Unfortunately, with the research design adopted by Weston et al., ${ }^{[55]}$ it is not possible to discriminate between the effectiveness of pitch (refereeing specific) and track training.

Recently, Impellizzeri et al. ${ }^{[88]}$ showed that soccer-specific training in the form of small-sided games carried out at $90-95 \%$ of $\mathrm{HR}_{\max }(4 \times 4$ minutes with 3 minutes of active recovery) were as effective as the Helgerud et al. ${ }^{[46]}$ protocol in improving aerobic fitness and match performance in soccer players. This finding suggests that specific or generic training may be equally effective in improving aerobic fitness and sport-specific performance when performed at a selected work intensity (i.e. 90-95\% of $\left.\mathrm{HR}_{\max }\right)$. As a result, aerobic fitness may be increased using diversified training means as long as the HR remains within 90-95\% of the individual maximal for at least 16 minutes per session and for two sessions a week. This means that referees could be involved in playing small-sided games as a valid fitness option.

However, Impellizzeri et al. ${ }^{[88]}$ found that training at $90-95 \%$ of $H_{R_{\max }}$ has differing effects on the physiological components of aerobic fitness. In this regard, after the summer intermission, the use of the Helgerud et al. ${ }^{[46]}$ method for 4 weeks significantly affected all the components of aerobic fitness. However, continuing in the $90-95 \% \mathrm{HR}_{\max }$ zone for a further 8 weeks during the competitive season, only resulted in improvements in the speed at anaerobic threshold (+5\%). In light of the results of Impellizzeri et al., ${ }^{[8]}$ it seems that sustained use of this training mode (i.e. $90-95 \%$ of $\mathrm{HR}_{\max }$ ) causes mainly adaptations in lactate/anaerobic threshold.

Other authors ${ }^{[89]}$ assume that the closer the exercise intensity to $\dot{\mathrm{VO}}_{2 \max }$ the higher the training response, suggesting training intensity >90-95\% $\mathrm{HR}_{\text {max }}$. As exercise at $\dot{\mathrm{V}}_{2 \text { max }}$ can be sustained for no more than 5-7 minutes in trained individuals, ${ }^{\text {[90] }}$ the use of short-interval running bouts (15-30 seconds, work rest ratio $1: 1)^{[89,91]}$ may be useful to induce improvement in $\dot{\mathrm{V}}_{2}$ max and aerobic fitness. The interest for soccer refereeing lies in that short interval training may simulate what referees usually experience during actual match-play in terms of the work-rest ratio ${ }^{[26,27]}$ while training to increase maximal aerobic power. In this regard, interval training could be implemented in the form of a circuit, in line with that reported by Hoff et al. ${ }^{[92]}$ and Chamari et al. ${ }^{[93]}$ for soccer players and by D'Ottavio ${ }^{[94]}$ for elite soccer referees.

As elite soccer referees are usually $>35$ years old with impairment of physical performance reported as being significant from 30 years of age, ${ }^{[95]}$ the possible age-related effect of training on physiological adaptation in soccer referees is therefore of particular interest. In this regard, it has been recently reported that aerobic and anaerobic performance considered as distance covered during the 12-minute running test, vertical jump and $200 \mathrm{~m}$ sprint, respectively, experience age-related performance decrements. ${ }^{[79]}$ These findings suggest once again that age impairs physical performance in humans and probably referees should train more as age progresses. A recent study ${ }^{[96]}$ showed that training adaptations are similar in old and young people with respect to $\dot{\mathrm{V}} \mathrm{O}_{2 \text { max }}$ and work performed at exhaustion. Training load differences and or genetic factors may cause different performance.

Although less relevant to match physical performance, ${ }^{[31]}$ anaerobic fitness should also be addressed in elite-level soccer referees. In fact, strength and power have been shown to decrease progressively across ages ${ }^{[95]}$ and this may negatively affect sprint ability and/or muscular efficiency. The significant age-related decrements observed in Italian elite soccer referees in standing vertical-jump 
and short-term sprint (approximately 30 seconds) performance ${ }^{[79]}$ support the notion that proper strength-power training should be provided to referees. In this regard, interval training (12-15 bouts of 15 seconds with 15 seconds of passive recovery) performed at $120 \%$ of speed at $\dot{\mathrm{VO}}_{2 m a x}$ in association with sprint training $(12-15 \times 40 \mathrm{~m}$ all-out bouts with 30 seconds of passive recovery) has been recently reported to improve aerobic performance and $40 \mathrm{~m}$ sprint performance. ${ }^{[97]}$

\section{Mental Performance}

Field refereeing should be regarded as a challenging role in soccer as the physical strain is superimposed on top of mental performance. Helsen and Bultynck $^{[18]}$ quantified the perceptual-cognitive workload of international games (final round of the Euro 2000 Championship) counting the number of observable decisions made by a referee. The results of the Helsen and Bultynck ${ }^{[18]}$ research showed that an international-level referee undertakes about 137 observable decisions of which $>60 \%$ are taken through communication with the assistant referees and/or the fourth official. Interestingly, during the second half, added-time decisions were twice those taken after the end of the first half, showing that the perceptual-cognitive workload progresses across the duration of the match. Included in the perceptualcognitive workload category are also the non-observable decisions, with the total number of decisions being raised to approximately 200, which is 3-4 decisions per minute. ${ }^{[18]}$ Given that, it may be argued that referees are constantly under mental stress during the match.

Although the physiological performance of elite soccer referees has received progressive attention from sport physiologists, the effect of physical stress on decision-making has not yet been investigated in detail. Furthermore, the available research ${ }^{[98]}$ utilised laboratory simulations (continuous $2 \times 45$ minutes of treadmill running at match intensity) that lack external validity as they do not depict what actually takes place during a competitive match (i.e. intermittent high-intensity exercise) played in a crowded stadium. Additionally, no research on this issue was carried out utilising referees as subjects. ${ }^{[98]}$ Thus, the reported no effect on the decisionmaking reported by Marriot et al. ${ }^{[98]}$ in their study may not reflect what actually takes place during competition in soccer referees.

Although improved physical fitness has been shown to positively affect match physical performance ${ }^{[27,31,99]}$ and optimal positioning, ${ }^{[29]}$ during a match it is not clear if exercise capacity actually may decrease the likelihood of incurring judgment errors during the game. A valuable attempt in this direction has been made by Verheijen and colleagues, ${ }^{[100]}$ who studied the effect of activity mode over decisions in three top-level soccer referees during youth soccer games. Those preliminary results showed that more correct decisions were taken moving at slower velocity compared with incorrect decisions $(2 \pm 1.04$ vs $4.17 \pm 1.98 \mathrm{~m} /$ second, respectively; $\mathrm{p}<0.001$ ). Unexpectedly, correct decisions were taken at a significantly longer distance from the infringement compared with incorrect judgments $(12.68 \pm 5.68 \mathrm{vs}$ $17.74 \pm 7.58 \mathrm{~m}$, respectively; $\mathrm{p}<0.001)$.

\section{Conclusions and Future Research}

The role of the referee is far from marginal in the economy of soccer as very often and particularly in professional soccer a wrong judgment may have profound implications on the outcome of the game. Recent studies have shown that during a competitive match an elite soccer referee may cover $9-13 \mathrm{~km}$ attaining approximately $85-90 \%$ and approximately $70-80 \%$ of $\mathrm{HR}_{\max }$ and $\dot{\mathrm{VO}}_{2 \max }$, respectively. Blood lactate concentration has been reported to be in the range of 4-5 mmol/L; however, during competitive matches, blood lactate concentration as high as 14 $\mathrm{mmol} / \mathrm{L}$ have been observed. Given that referees are also required to keep up with the game and have very little scope for rest periods, it is clear that refereeing (especially at the elite level) is as demanding, if not more demanding, than playing.

In our opinion, future research should strive to study the effects of physical fitness (aerobic and anaerobic fitness) and exercise mode (i.e. low- vs high-intensity activities) on competitive stress and decision-making during actual match play. 
Furthermore, in light of the very limited research on cognitive functioning, laboratory and field-based research should be implemented using appropriate game-simulation protocols. This will enable the examination of the effect of exercise on a referee's decision-making in conditions that demand exceptional judgment based on fast-changing visual scenes. Additionally, the effect of age upon the referees physical and cognitive match performance is worth examining. In this regard, an ergonometric approach $^{[8]}$ integrating perceptual-cognitive and physical performance optimisation may prove useful in gaining knowledge over soccer referee performance. This is with the aim to improve referees performance while progressively developing refereeing as a professional activity, and ultimately for the good of the game.

\section{Acknowledgements}

No sources of funding were used to assist in the preparation of this review. The authors have no conflicts of interest that are directly relevant to the content of this review.

\section{References}

1. Peiser B, Minten, J. Soccer violence. In: Reilly T, Williams AM, editors. In: Science and soccer. 2nd ed. London: Routledge, 2003: $230-41$

2. Reilly T. Energetics of high-intensity exercise (soccer) with particular reference to fatigue. J Sports Sci 1997; 15: 257-63

3. FIFA. Where it all began [online]. Available from URL: http:// www.fifa.com [Accessed 2002 Sep 9]

4. Reilly T, Gilbourne D. Science and football: a review of applied research in the football codes. J Sports Sci 2003; 21: 693-705

5. Stølen T, Chamari K, Castagna C, et al. Physiology of soccer: an update. Sports Med 2005; 35 (6): 501-36

6. Tumilty D. Physiological characteristics of elite soccer players. Sports Med 1993; 16: 80-96

7. Shephard RJ. Biology and medicine of soccer: an update. J Sports Sci 1999; 17: 757-86

8. Reilly T. An ergonomics model of the soccer training process. J Sports Sci 2005; 23 (6): 561-72

9. Mohr M, Krustrup P, Bangsbo J. Fatigue in soccer: a brief review. J Sports Sci 2005; 23 (6): 593-9

10. Impellizzeri FM, Rampinini E, Marcora SM. Physiological assessment of aerobic training in soccer. J Sports Sci 2005; 23 (6): 583-92

11. Ekblom B. Applied physiology of soccer. Sports Med 1986; 3 : 50-60

12. Svensson M, Drust B. Testing soccer players. J Sports Sci 2005; 23 (6): 601-18

13. Hoff J, Helgerud J. Endurance and strength training for soccer players: physiological considerations. Sports Med 2004; 34 (3): $165-80$
14. Hoff J. Training and testing physical capacities for elite soccer players. J Sports Sci 2005; 23 (6): 573-82

15. Abt GA, Dickson G, Mummery WK. Goal scoring patterns over the course of a match: an analysis of the Australian Nationa Soccer League. In: Spinks W, Reilly T, Murphy A, editors. Science and football IV. London: Routledge, 2002: 106-11

16. Eissmann HJ. The 23rd man: sport medical advice for football referees. Leipzig: Gersöne-Druck, 1996

17. Féderation Internationale de Football Association. Laws of the game 2005. Zurich: Féderation Internationale de Football Association, 2005

18. Helsen W, Bultynck JB. Physical and perceptual-cognitive demands of top-class refereeing in association football. J Sports Sci 2004; 22: 179-89

19. Bangsbo J. The physiology of soccer: with special reference to intense intermittent exercise. Acta Physiol Scand 1994; 151 Suppl. 619: 1-155

20. Bangsbo J. Time and motion characteristics of competitive soccer. Sci Football 1992; 6 (2): 34-40

21. Mohr M, Krustrup P, Bangsbo J. Match performance of highstandard soccer players with special reference to development of fatigue. J Sports Sci 2003; 21: 519-28

22. Castagna C, D'Ottavio S, Abt G. Activity profile of young soccer players during actual match play. J Strength Cond Res 2003; 17 (4): 775-80

23. Asami T, Togari H, Ohashi J. Analysis of movement patterns of referees during soccer matches. In: Reilly T, Lees A, Davids K, et al., editors. Science and football. London: E \& FN Spon, 1988: $341-5$

24. Johnston L, McNaughton L. The physiological requirements of soccer refereeing. Aust J Sci Med Sport 1994; 26 (3/4): 67-72

25. Catterall C, Reilly T, Atkinson G, et al. Analysis of work rate and heart rates of association football referees. Br J Sports Med 1993; 27: 153-6

26. D'Ottavio S, Castagna C. Analysis of match activities in elite soccer referees during actual match play. J Strength Cond Res 2001; 15 (2): 167-71

27. Krustrup P, Bangsbo J. Physiological demands of top-class soccer refereeing in relation to physical capacity: effect of intense intermittent exercise training. J Sports Sci 2001; 19: 881-91

28. Castagna C, Abt G, D'Ottavio S. Activity profile of international-level soccer referees during competitive matches. J Strength Cond Res 2004; 18 (3): 486-90

29. Harley RA, Tozer K, Doust J. An analysis of movement patterns and physiological strain in relation to optimal positioning of Association Football referees. In: Spinks W, Reilly T, Murphy A, editors. Science and football IV. London: Routledge, 2002: 137-43

30. Castagna C, D'Ottavio S. Effect of maximal aerobic power on match performance in elite soccer referees. J Strength Cond Res 2001; 15 (4): 420-5

31. Castagna C, Abt G, D'Ottavio S. Relation between fitness tests and match performance in elite Italian soccer referees. J Strength Cond Res 2002; 16 (2): 231-5

32. Castagna C, Abt G, D'Ottavio S. The relationship between selected blood lactate thresholds and match performance in elite soccer referees. J Strength Cond Res 2002; 16 (4): 623-7

33. Reilly T. Motion analysis and physiological demands. In: Reilly T, Williams AM, editors. Science and soccer. London: E \& FN Spon, 2003: 59-72

34. Bangsbo J, Norregaard L, Thorso F. Activity profile of competition soccer. Can J Sport Sci 1991; 16 (2): 110-6 
35. Williams AM, Lee D, Reilly T. A quantitative analysis of matches played in the 1991-92 and 1997-98 seasons. London: The Football Association, 1999

36. D'Ottavio S, Castagna C. Physiological load imposed on elite soccer referees during actual match play. J Sports Med Phys Fitness 2001; 41 (1): 27-32

37. D'Ottavio S, Castagna C. Physiological aspects of soccer refereeing. In: Spinks W, Reilly T, Murphy A, editors. Science and football IV. London: Routledge, 2002: 144-50

38. Wisløff U, Helgerud J, Hoff J. Strength and endurance of elite soccer players. Med Sci Sports Exerc 1998; 30 (3): 462-7

39. Weltman A. The blood lactate response to exercise. Champaign (IL): Human Kinetics, 1995

40. Reilly T, Bowen T. Exertional cost of changes in directional modes of running. Percept Motor Skills 1984; 58: 49-50

41. Harley RA, Banks R, Doust J. The development and evaluation of a task specific fitness test for association football referees. In: Spinks W, Reilly T, Murphy A, editors. Science and football IV. London: Routledge, 2002: 76-80

42. Castagna $\mathrm{C}$, Abt $\mathrm{G}$. Intermatch variation of match activity in elite Italian soccer referees. J Strength Cond Res 2003; 17 (2): 388-92

43. Ohashi J, Togari H, Isokawa M, et al. Measuring movement speeds and distances covered during soccer match-play. In: Reilly T, Lees A, Davids K, et al., editors. Science and football. London: E \& FN Spon, 1988: 329-33

44. Reilly T. Motion analysis and physiological demands. In: Reilly T, Williams AM, editors. Science and soccer. London: E \& FN Spon, 2003: 59-72

45. Krustrup P, Mohr M, Bangsbo J. Activity profile and physiological demands of top-class soccer assistant refereeing in relation to training status. J Sports Sci 2002; 20: 861-71

46. Helgerud J, Engen LC, Wisløff U, et al. Aerobic endurance training improves soccer performance. Med Sci Sports Exerc 2001; 33 (11): 1925-31

47. Weston M, Brewer J. A study of the physiological demands of soccer refereeing. J Sports Sci 2002; 20: 59-60

48. Snyder AC, Kuipers H, Cheng B, et al. Overtraining following intensified training with normal muscle glycogen. Med Sci Sports Exerc 1995; 27 (7): 1063-70

49. Urhausen A, Gabriel HH, Weiler B, et al. Ergometric and psychological findings during overtraining: a long-term follow-up study in endurance athletes. Int J Sports Med 1998; 19 (2): $114-20$

50. Lehmann M, Dickhuth HH, Gendrisch G, et al. Training-overtraining: a prospective, experimental study with experienced middle-and long-distance runners. Int J Sports Med 1991; 12 (5): 444-52

51. Jeukendrup AE, Hesselink MKC, Snyder AC, et al. Physiological changes in male competitive cyclists after two weeks of intensified training. Int J Sports Med 1992; 13: 534-41

52. Hooper SL, Mackinnon LT, Howard A. Physiological and psychometric variables for monitoring recovery during tapering for major competition. Med Sci Sports Exerc 1999; 31 (8) 1205-10

53. Bosquet L, Léger L, Legros O. Blood lactate response to overtraining in male endurance athletes. European J Appl Physiol 2001; 84: 107-14

54. Halson SL, Bridge MW, Meeusen R, et al. Time course of performance changes and fatigue markers during intensified training in trained cyclists. J Appl Physiol 2002; 93 (3): 947-56
55. Weston M, Helsen W, MacMahon C, et al. The impact of specific high-intensity training sessions on football referees' fitness levels. Am J Sport Med 2004; 32 (1 Suppl.): 54-61s

56. Achten J, Jeukendrup A. Heart rate monitoring: applications and limitations. Sports Med 2003; 33 (7): 517-38

57. Åstrand P, Rodahl K. Textbook of Work physiology: physiological bases of exercise. 3rd ed. New York: McGraw-Hill, 1986

58. Castagna C, Abt G, D'Ottavio S. Competitive-level differences in Yo-Yo intermittent recovery and 12 min run test performance in soccer referees. J Strength Cond Res 2005; 19 (4) 805-9

59. Rodriguez FA, Iglesias X. The energy cost of soccer: telemetric oxygen uptake measurements versus heart rate- $\dot{\mathrm{V}} \mathrm{O}_{2}$ estimations [abstract]. J Sports Sci 1998; (16): 484-5

60. Smith M, Clarke G, Hale T, et al. Blood lactate levels in college soccer players during match-play. In: Reilly T, Clarys J, Stibbe A, editors. Science and football II. London: E \& FN Spon, 1993: $129-34$

61. Saltin B, Costill DL. Fluid and electrolyte balance during prolonged exercise. New York: Macmillan, 1988

62. Sawka MN, Pandolf KB. Effect of body water loss on physiological functioning and exercise performance. In: Gisolfi CV, Lamb DR, editors. Fluid homeostasis during exercise. Carmel (IN): Cooper, 1990: 1-38

63. Coyle EF. Fluid and fuel intake during exercise. J Sports Sci 2004; 22: 39-55

64. Maughan RJ, Leiper JB. Fluid replacement requirements in soccer. J Sports Sci 1994; 12: S29-34

65. Gopinathan PM, Pichan G, Sharma VM. Role of dehydration in heat stress-induced variations in mental performance. Arch Environ Health 1988; 43 (1): 15-7

66. Davies PD, Cooke CB, King RFGJ. Fluid loss and replacement in English premier league football players. In: Reilly T, Bangsbo J, Hughes MA, editors. Science and football III. London: E. \& F.N. Spon, 1996: 54-9

67. Mustafa KY, Mahmoud ED. Evaporative water loss in African soccer players. J Sports Med Phys Fitness 1979; 19: 181-3

68. McGregor SJ, Nicholas CW, Lakomy HKA, et al. The influence of intermittent high-intensity shuttle running and fluid ingestion on the performance of a soccer skill. J Sports Sci 1999; 17: 895-903

69. Nicholas CW, Nuttall FE, Williams C. The Loughborough Intermittent Shuttle Test: a field test that simulates the activity pattern of soccer. J Sports Sci 2000; 18: 97-104

70. Da Silva AI, Fernandez R. Dehydration of football referees during a match. Br J Sports Med 2003; 37: 502-6

71. Garganta J, Maia J, Basto F. Analysis of goal-scoring patterns in European top level soccer teams. In: Reilly T, Bangsbo J, Hughes M, editors. Science and football III. London: E \& FN Spon, 1997: 246-50

72. Grehaigne J-F. Time distribution of goals in soccer: some championships and the 1998 World Cup. In: Hughes M, Tavarez F, editors. IV World Congress of Notational Analysis of Sport Porto (Portugal): Centre for Team Sports Studies, Faculty of Sport Sciences and Physical Education, University of Porto, 1998: 41-50

73. Reilly T, Lewis W. Effects of carbohydrate feeding on mental functions during sustained physical work. In: Brown ID, Goldsmith R, Coombes K, et al., editors. Ergonimics international 85. London: Taylor and Francis, 1985: 700-2

74. Reilly T. Environmental stress. In: Reilly T, Williams M, editor. Science and soccer. Routledge: London, 2003: 165-84 
75. Rontoyannis GP, Stalikas A, Sarros G, et al. Medical, morphological and functional aspects of Greek football referees. J Sports Med Phys Fitness 1998; 38: 208-14

76. Barbanti V, Bergson P. Anthropometric and physical capacity characteristics of Brazilian soccer referees and referee assistants. In: Tavarez F, editor. IV World Congress on Notational Analysis of sport. Porto (Portugal): FCDEF-Porto University, 1998: 35

77. Eissmann HJ, D’Hooghe M. Sports medical examinations. In: Eissmann HJ, editor. The 23rd man: sports medical advice for football referees. Leipzig: Gersöne-Druck, 1996: 7-19

78. Wilmore JH, Costill DL. Physiology of sport and exercise. 2nd ed. Champaign (IL): Human Kinetics, 1999

79. Castagna C, Abt G, D'Ottavio S, et al. Age-related effects on fitness performance in elite-level soccer referees. J Strength Conditioning Res 2005; 19 (4): 785-90

80. Bangsbo J, Mohr M, Krustrup P. Physical capacity and match performance of top-class referees in relation to age. J Sports Sci 2004; 22 (6): 485-593

81. Reilly T, Bangsbo J, Franks A. Anthropometric and physiological predispositions for elite soccer. J Sports Sci 2000; 18 669-83

82. Wisløff U, Castagna C, Helgerud J, et al. Maximal squat strength is strongly correlated to sprint-performance and vertical jump height in elite soccer players. Br J Sports Med 2004; 38: $285-8$

83. Bosco C, Komi PV. Influence of aging on the mechanical behaviour of leg extensor muscles. Eur J Appl Physiol 1980; (45): 209-19

84. Ramsbottom R, Brewer J, Williams C. A progressive shuttle run test to estimate maximal oxygen uptake. Br J Sports Med 1988; 22 (4): 141-4

85. Leger LA, Mercier D, Gadoury C, et al. The multistage 20 metre shuttle run test for aerobic fitness. J Sports Sci 1988; 6 (2): 93-101

86. Krustrup P, Mohr M, Amstrup T, et al. The Yo-Yo Intermittent Recovery Test: physiological response, reliability, and validity. Med Sci Sports Exerc 2003; 35 (4): 697-705

87. Pate RR, Kriska A. Physiological basis of the sex difference in cardiorespiratory endurance. Sports Med 1984; 1 (2): 87-98

88. Impellizzeri FM, Marcora SM, Castagna C, et al. Physiological and performance effects of generic versus specific aerobic training in soccer players. Int J Sports Med 2006; 27 (6): 483-92

89. Billat VL, Slawinski J, Bocquet V, et al. Intermittent runs at the velocity associated with maximal oxygen uptake enables sub- jects to remain at maximal oxygen uptake for a longer time than intense but submaximal runs. Eur J Appl Physiol 2000; 81: $188-96$

90. Billat V, Koralsztein JP. Significance of the velocity at $\dot{V}_{2}$ max and time to exhaustion at this velocity. Sports Med 1996; 22: 90-180

91. Billat VL, Slawinks J, Bocquet V, et al. Very short (15s - 15s) interval-training around the critical velocity allows middleaged runners to maintain $\dot{\mathrm{VO}}_{2 \max }$ for 14 minutes. Int J Sports Med 2001; 22: 201-8

92. Hoff J, Wisloff U, Engen LC, et al. Soccer specific aerobic endurance training. Br J Sports Med 2002; 36 (3): 218-21

93. Chamari K, Hachana Y, Kaouech F, et al. Endurance training and testing with the ball in young elite soccer players. Br J Sports Med 2005; 39 (1): 24-8

94. D'Ottavio S. Un allenamento che vale una partita. Il Nuovo Calcio 1993; 10: 40-2

95. Taylor AH, Cable NT, Faulkner G, et al. Physical activity and older adults: a review of health benefits and the effectiveness of interventions. J Sports Sci 2004; 22 (8): 703-25

96. Lawrenson L, Hoff J, Richardson RS. Aging attenuates vascular and metabolic plasticity but does not limit improvement in muscle $\dot{V O}_{2 \max }$. Am J Physiol Heart Circ Physiol 2004; 286 (4): $1565-72$

97. Dupont G, Akakpo K, Berthoin S. The effect of in-season, highintensity interval training in soccer players. J Strength Cond Res 2004; 18 (3): 584-9

98. Marriott J, Reilly T, Miles A. The effect of physiological stress on cognitive performance in a simulation of soccer, In: Reilly T, Clarys J, Stibbe A, editors. Science and football II. London: E \& FN Spon, 1993: 261-4

99. D'Ottavio S, Annino G, Padua E, et al. Effect of $\dot{V}_{2} \max$ level on repeated sprint ability in basketball players. In: Van Praagh E, Coudert J, Fellmann N, et al., editors. 9th Annual Congress European College of Sport Science. Clermont-Ferrand: UFR STAPS Clermont-Ferrand, 2004: 307

100. Verheijen R, Oudejans R, Beek PJ, et al. Factors affecting decision making of soccer referees. In: Spinks W, editor. Fourth Word Congress of Science and Football. Sydney: University of Technology, Sydney, 1999: 28

Correspondence: Carlo Castagna, Via Sparapani 30, 60131 Ancona, Italy.

E-mail: castagnac@libero.it 